\title{
Étude de l'incidence des quantités d'aliments ingérées, et de la période du nycthémère sur les niveaux moyens de l'ammoniaque ruminale et de l'urémie chez la vache frisonne tarie
}

\author{
Dehareng, J-M Godeau, A Hubsch, F Beaussart, \\ P Debue, B Ndibualonji, A Huybens
}

Centre de recherche de I'IRSIA, faculté de médecine vétérinaire, rue des vétérinaires, service de biochimie normale et pathologique, 45, B-1070 Bruxelles, Belgique

\begin{abstract}
Summary - The average levels of ruminal ammonia and of uremia in Friesian dry cows : a study on the influence of the feeding level and of the nycthemeral period of measurement. A continuous collection ( $1 \mathrm{~d}$; Dehareng et al, 1988) of rumen liquor (for $\mathrm{NH}_{3}$ ) and blood (for uremia) was performed on 4 dry Friesian cows fed (2 times / d) at low or high level (hay + concentrate diet; 3.7 and $8.8 \mathrm{~kg}$ dry matter/d). The feeding level had no incidence on the daily average values but the morning (from $06 \mathrm{~h}$ to $12 \mathrm{~h}$ ) means always exhibited the highest $(P<0.05)$ nycthemeral values. Ruminal $\mathrm{NH}_{3}$ and uremia were only related $(P<0.05)$ in the period before the morning meal $(00-06 \mathrm{~h})$.
\end{abstract}

L'incidence des quantités d'aliments ingérées et de la période du nycthémère sur l'ammoniaque du rumen et sur l'urémie a été étudiée chez la vache Frisonne tarie au moyen de techniques de prélèvement en continu (Dehareng et al, 1988) des liquides physiologiques.

Matériel et méthodes - Quatre vaches taries, non gravides et munies d'une canule ruminale, ont été alimentées en 2 repas quotidiens égaux (06 h $15-15$ h 30 ), avec du foin $F$ (prairie naturelle) distribué avant un aliment concentré $C$ du commerce. La ration journalière a d'abord été constituée de $2 \mathrm{~kg}$ de $F$ et de $2 \mathrm{~kg}$ de $C$ (ration $1: 3,7 \mathrm{~kg} \mathrm{MS}$; $87 \mathrm{~g} \mathrm{~N} ; 73 \mathrm{MJ} \mathrm{EB}$; apports théoriquement déficitaires en énergie et suffisants en $N$ ), puis de $5 \mathrm{~kg}$ de chaque aliment (ration 2: 8,8 kg MS; 192 g N; 177 MJ EB; apports excédentaires en énergie et en $\mathrm{N}$ ). Après l'adaptation (4 semaines) à chaque régime, les vaches ont été pesées ( 1 fois à $09 \mathrm{~h}$ ) et soumises à une mesure $(5 j)$ de la digestibilité apparente du $\mathrm{N}$ alimentaire puis à un prélèvement en continu (24 h) de liquide ruminal (mesure du $\mathrm{NH}_{3}$ ) et de sang veineux (dosage de l'urée). Les valeurs moyennes des paramètres étudiés ont été établies sur l'ensemble du nycthémère et par période de $6 \mathrm{~h}$, la première de celles-ci débutant à minuit.

Résultats et discussion - La digestibilité apparente du $\mathrm{N}$ alimentaire a été similaire $(P>0,05)$ pour les 2 régimes étudiés : $53,3 \pm 6,9 \%$ (ration 2) versus $57,8 \pm 8,0 \%$ (ration 1). En conséquence, la ration 2 a apporté 2 fois plus d'azote digestible $(103 \pm 13$ vs $50 \pm$ $7 \mathrm{~g} / \mathrm{j} ; P<0,05)$ que le régime 1 , ce qui, en sus de l'accroissement des apports énergétiques et du poids du contenu digestif, explique l'augmentation du poids vif moyen des animaux (533 \pm 36 vs $490 \pm 24 \mathrm{~kg} ; P<0,05)$.

Relativement à la ration 1 , le régime 2 s'est également accompagné d'une tendance $(P<0,05)$ à l'accroissement de la teneur journalière moyenne en $\mathrm{NH}_{3}$ du liquide ruminal (148 \pm 53 vs $130 \pm$ $16 \mathrm{mg} / \mathrm{l}$ ) et, contrairement à la bibliographie, à la diminution du niveau journalier moyen de l'urémie $282 \pm 59$ vs $341 \pm 92 \mathrm{mg} / \mathrm{l})$. En effet, l'augmentation de l'apport azoté de rations isoénergétiques (Huntington, 1989) ou non (Hennessy et Nolan, 1988) induit toujours une augmentation du niveau de l'urémie. Toutefois, l'urémie observée avec la ration 1 (pauvre en apports énergétiques) doit être induite par une augmentation de la néoglucogenèse acides aminés-dépendante pour couvrir les besoins énergétiques (Champredon et al, 1987). En revanche, le régime 2 couvre ces besoins mais il ne peut 
réduire sensiblement le niveau de l'urémie car celle-ci est soutenue par un apport d'azote exogène au-dessus des besoins (Tagari et al, 1964). L'absence d'effet statistique des quantités ingérées sur les paramètres du rumen et du sang peut être partiellement expliquée par la similitude du rapport $\mathrm{N}$ total/énergie des 2 régimes (Ropstad et al, 1989).

Par ailleurs, pour chaque régime étudié, les mesures matinales (06 $\mathrm{h}-$ midi) de la concentration en $\mathrm{NH}_{3}$ (fig 1) dans le rumen ont, en moyenne, été supérieures $(P<0,05)$ à celles observées pendant la fin de la nuit (minuit - $06 \mathrm{~h}$ ) et pendant l'après-midi (midi - $18 \mathrm{~h}$ ). Ces observations ne peuvent pas s'expliquer uniquement par l'incidence de la dégradation des aliments du repas du matin puisque les quantités ingérées lors de chaque repas furent identiques. De même, l'urémie mesurée au cours de la matinée (06 h - midi) présente, pour chaque ration étudiée, les valeurs nycthémérales les plus élevées (fig 1) et, avec le régime 1 , elle est statistiquement différente des autres périodes. Pour chaque ration étudiée et au sein de chaque période du nycthémère comme pour l'ensemble de ce dernier, aucune relation linéaire interindividuelle

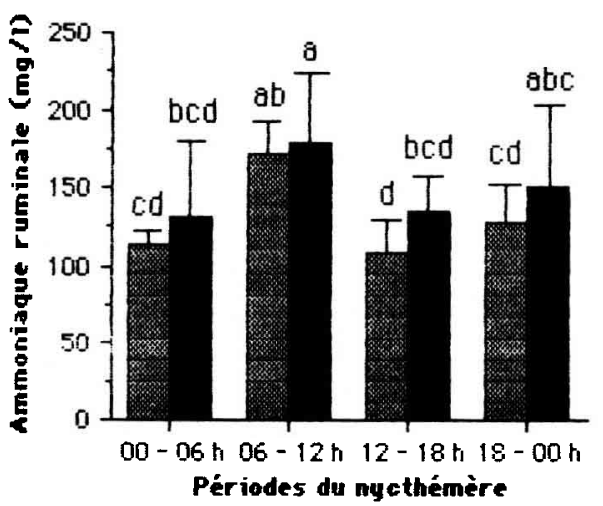

n'a pu être établie entre les niveaux moyens de l'urémie ( $U, \mathrm{mg} / \mathrm{l}$ ) et ceux de l'ammoniaque (concentration $C$, $\mathrm{mg} / \mathrm{l}$ ), à l'exception de la seule période nocturne (minuit - $06 \mathrm{~h}$ ) enregistrée pour le régime $2: U=103+1,4 \mathrm{C}$ $(R=0,996 ; n=4 ; P<0,01)$, suggérant ainsi l'absence d'une relation simple entre le $\mathrm{NH}_{3}$ ruminal et l'urémie en périodes postprandiales mais non en période préprandiale matinale.

En conclusion, si les valeurs du $\mathrm{NH}_{3}$ ruminal et de l'urémie n'ont pas été affectées par les quantités d'aliments ingérées lors de cet essai, elles sont toutefois dépendantes du moment du nycthémère au cours duquel elles sont mesurées, la période matinale $(06 \mathrm{~h}$ midi) étant caractérisée par les valeurs les plus élevées.

Champredon C, Rémond B, Patureau-Miraud D (1987) Reprod Nutr Dév 27, 301-302

Dehareng D, Huybens A, Godeau J-M (1988) Med Fac Landbouww Rijksuniv Gent 53 (4a), 1831-1838

Hennessy DW, Nolan JV (1988) Aust J Agric Res 39, 215-223

Huntington GB (1989) Can J Anim Sci 69, 215-223

Ropstad E, Vik-Mo L, Refsdal AO (1989) Acta Vet Scand 30, 199-208

Tagari H, Dror Y, Ascarelli I, Bondi A (1964) Br J Nutr 18, 333-356

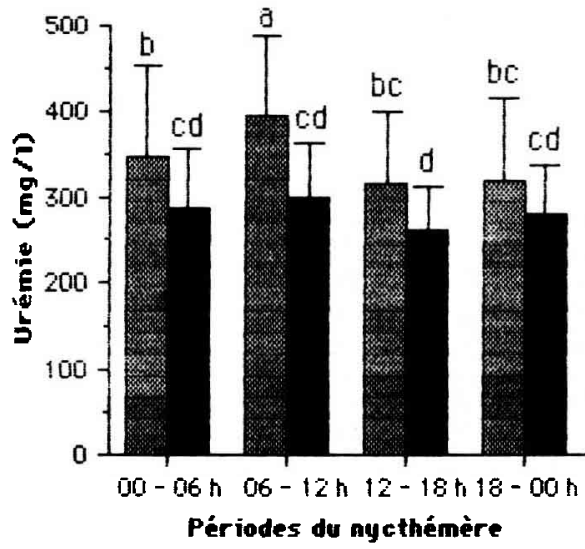

Fig 1. Évolutions moyennes (+ déviation standard) de la teneur en ammoniaque ruminale et de l'urémie (rectangles gris: ration 1; rectangles noirs: ration 2). a, b, c, d: les moyennes ne présentant aucune lettre indicée commune diffèrent statistiquement $(P<0,05)$. 